Technical Report

13 March 2007

\title{
Hermite polynomials on the plane
}

\author{
Shayne Waldron
}

Department of Mathematics, University of Auckland, Private Bag 92019, Auckland, New Zealand e-mail: waldron@math.auckland.ac.nz (http:www.math.auckland.ac.nz/ ${ }^{\sim}$ waldron)

\begin{abstract}
The space $\mathcal{P}_{n}$ of bivariate generalised Hermite polynomials of degree $n$ is invariant under rotations. We exploit this symmetry to construct an orthonormal basis for $\mathcal{P}_{n}$ which consists of the rotations of a single polynomial through the angles $\frac{\ell \pi}{n+1}, \ell=0, \ldots n$. Thus we obtain an orthogonal expansion which retains as much of the symmetry of $\mathcal{P}_{n}$ as is possible. Indeed we show that a continuous version of this orthogonal expansion exists.
\end{abstract}

Key Words: bivariate Hermite polynomials, Laguerre polynomials, Zernike polynomials, quadrature for trigonometric polynomials, representation theory, orthogonal expansions

AMS (MOS) Subject Classifications: primary 33C45, 33D50, secondary 06B15, $42 \mathrm{C} 15$ 


\section{Introduction}

Here we consider the space $\mathcal{P}_{n}$ of generalised Hermite polynomials in the plane $\mathbb{R}^{2}$. This $n+1$ dimensional space consists of all bivariate polynomials of degree $n$ which are orthogonal to all polynomials of degree $<n$ with respect to the inner product

$$
\begin{aligned}
\langle f, g\rangle & :=\int_{\mathbb{R}^{2}} f(x) g(x)\|x\|^{2 \beta} e^{-\|x\|^{2}} d x \\
& =\int_{0}^{2 \pi} \int_{0}^{\infty}(f g)(r \cos \theta, r \sin \theta) r^{2 \beta} e^{-r^{2}} r d r d \theta, \quad \beta \geq 0
\end{aligned}
$$

Our calculations can be extended to any radially symmetric weight.

Let $R_{\theta}: \mathbb{R}^{2} \rightarrow \mathbb{R}^{2}$ denote rotation through the angle $\theta$, i.e.,

$$
R_{\theta}(x, y):=\left(\begin{array}{cc}
\cos \theta & -\sin \theta \\
\sin \theta & \cos \theta
\end{array}\right)\left(\begin{array}{l}
x \\
y
\end{array}\right)=\left(\begin{array}{c}
x \cos \theta-y \sin \theta \\
x \sin \theta+y \cos \theta
\end{array}\right)
$$

The group of rotations of the plane (which are symmetries of the weight)

$$
\mathrm{SO}(2)=\left\{R_{\theta}: 0 \leq \theta<2 \pi\right\}
$$

acts naturally on functions $\mathbb{R}^{2} \rightarrow \mathbb{R}$ or $\mathbb{R}^{2} \rightarrow \mathbb{C}$ via

$$
R_{\theta} f:=f \circ R_{\theta}
$$

The space $\mathcal{P}_{n}$ is invariant under $\mathrm{SO}(2)$, i.e.,

$$
R_{\theta} f \in \mathcal{P}_{n}, \quad \forall f \in \mathcal{P}_{n},
$$

and satisfies the condition

$$
R_{\pi} f=(-1)^{n} f, \quad f \in \mathcal{P}_{n} .
$$

We will construct an orthonormal basis for $\mathcal{P}_{n}$ which consists of the rotations of a single polynomial through the angles $\frac{\ell \pi}{n+1}, \ell=0, \ldots, n$. In view of (1.2) and (1.3), this basis retains as much of the symmetry of $\mathcal{P}_{n}$ as is possible, and so one might argue has the simplest possible form.

There are two known orthonormal bases for multiple Hermite polynomials. One is obtained by factorising the weight, and the other by taking spherical harmonic factors of the orthogonal polynomials.

\section{Factorisation of the weight}

If $\beta=0$, then (and only then) the Hermite weight function factors

$$
e^{-\left(x^{2}+y^{2}\right)}=e^{-x^{2}} e^{-y^{2}}
$$


so that an orthogonal basis for $\mathcal{P}_{n}$ is given by the polynomials

$$
(x, y) \mapsto H_{j}(x) H_{n-j}(y), \quad 0 \leq j \leq n,
$$

where $H_{n}$ denotes the univariate Hermite polynomials, which are given by

$$
H_{n}(x):=(-1)^{n} e^{x^{2}} \frac{d^{n}}{d x^{n}} e^{-x^{2}}, \quad \int_{\mathbb{R}}\left(H_{n}(x)\right)^{2} e^{-x^{2}} d x=\sqrt{\pi} 2^{n} n ! .
$$

In [W01] the polynomials (1.4) are generalised to a biorthogonal system depending on a matrix parameter $U$ (which reduces to the orthogonal system when $U$ is the identity).

\section{Spherical harmonics}

It is convenient to allow the orthogonal polynomials in $\mathcal{P}_{n}$ to have complex coefficients, and at times replace the cartesian coordinates $x$ and $y$ by $z$ and $\bar{z}$, where $z:=x+i y$. We also allow the formula for a polynomial (in either system) to appear in place of the function in the inner product and the integral defining it, e.g., by integrating the polar form, we have

$$
\left\langle z^{j} \bar{z}^{k}, 1\right\rangle=\int_{\mathbb{R}^{2}} z^{j} \bar{z}^{k}|z|^{2 \beta} e^{-|z|^{2}}= \begin{cases}0, & j \neq k \\ m_{j}, & j=k\end{cases}
$$

where

$$
\begin{aligned}
m_{j}: & =\int_{\mathbb{R}^{2}}|z|^{2 j}|z|^{2 \beta} e^{-|z|^{2}}=2 \pi \int_{0}^{\infty} r^{2 \beta+2 j+1} e^{-r^{2}} d r \\
& =\pi \int_{0}^{\infty} t^{\beta+j} e^{-t} d t=\pi \Gamma(\beta+j+1)>0, \quad j=0,1, \ldots
\end{aligned}
$$

Thus the orthogonal projection of polynomials

$$
z \mapsto z^{j} \bar{z}^{n-j}, \quad 0 \leq j \leq n
$$

onto $\mathcal{P}_{n}$ forms an orthogonal basis for $\mathcal{P}_{n}$. We will refer to these and related polynomials as (generalised) Zernike polynomials, after the analoguous orthogonal polynomials on the disc, see, e.g., [DX01] for references. These factor

$$
z^{n-2 j} P_{j}\left(|z|^{2}\right), \quad j \geq n-j, \quad \bar{z}^{n-2 j} P_{j}\left(|z|^{2}\right), \quad n-j \geq j
$$

where $P_{j}$ is the (monic) polynomial of degree $j$ satisfying

$$
\left\langle z^{n-2 j} P_{j}\left(|z|^{2}\right), \bar{z}^{n-2 j} z^{s} \bar{z}^{s}\right\rangle=0, \quad 0 \leq s<j .
$$

By making the change of variables $x=r^{2}$, the condition (1.7) can be written

$$
\begin{aligned}
\left\langle z^{n-2 j} P_{j}\left(|z|^{2}\right), \bar{z}^{n-2 j} z^{s} \bar{z}^{s}\right\rangle & =\int_{\mathbb{R}^{2}} P_{j}\left(|z|^{2}\right)|z|^{2 s}|z|^{2 n-4 j}|z|^{2 \beta} e^{-|z|^{2}} \\
& =2 \pi \int_{0}^{\infty} P_{j}\left(r^{2}\right) r^{2 s} r^{2 n-4 j} r^{2 \beta} e^{-r^{2}} d r \\
& =2 \pi \int_{0}^{\infty} P_{j}(x) x^{s} x^{n-2 j} x^{\beta} e^{-x} \frac{d x}{2}=0
\end{aligned}
$$


so that $P_{j}$ is (up to normalisation) the Laguerre polynomial $P_{j}=L_{j}^{n-2 j+\beta}$, where $L_{n}^{\alpha}$ is given by

$$
L_{n}^{\alpha}(x):=\frac{1}{n !} x^{-\alpha} e^{x} \frac{d^{n}}{d x^{n}}\left(x^{n+\alpha} e^{-x}\right), \quad \int_{0}^{\infty}\left(L_{n}^{\alpha}(x)\right)^{2} x^{\alpha} e^{-x} d x=\frac{\Gamma(\alpha+n+1)}{n !} .
$$

A spherical harmonic of degree $n-2 j$ can be written in the form $z \mapsto \operatorname{Re}\left(\xi z^{n-2 j}\right)$, $\xi \in \mathbb{C}$. For this harmonic polynomial (1.6) gives

$$
\left.\left.\left.\operatorname{Re}\left(\xi z^{n-2 j}\right) P_{j}\left(|z|^{2}\right)\right)=\frac{1}{2} \xi z^{n-2 j} P_{j}\left(|z|^{2}\right)\right)+\frac{1}{2} \bar{\xi} \bar{z}^{n-2 j} P_{j}\left(|z|^{2}\right)\right)
$$

so that $z \mapsto \operatorname{Re}\left(\xi z^{n-2 j}\right) P_{j}\left(|z|^{2}\right)$ belongs to $\mathcal{P}_{n}(\operatorname{cf}[\mathrm{DX} 01: \S 2.3 .4])$.

We now construct our orthonormal basis in terms of these functions.

\section{The orthonormal basis}

First we give the normalisation and symmetry properties of the polynomials of the type (1.8).

Lemma 2.1. For $0 \leq j \leq \frac{n}{2}$ define polynomials $p_{j}: \mathbb{R}^{2} \rightarrow \mathbb{R}$ by

$p_{j}(x, y):=\frac{1}{\sqrt{2 \pi}} \frac{1}{\sqrt{1+\delta_{j, \frac{n}{2}}}} \frac{\sqrt{j !}}{\sqrt{\Gamma(\beta+n-j+1)}}\left(z^{n-2 j}+\bar{z}^{n-2 j}\right) L_{j}^{n-2 j+\beta}\left(|z|^{2}\right), \quad z:=x+i y$.

Then $p_{j} \in \mathcal{P}_{n}$, and for $\theta, \psi \in[0,2 \pi]$ and $0 \leq j, k \leq \frac{n}{2}$, we have

$$
\left\langle R_{\theta} p_{j}, R_{\psi} p_{k}\right\rangle= \begin{cases}0, & j \neq k \\ \cos ((n-2 j)(\theta-\psi)), & j=k\end{cases}
$$

Proof: The polynomials $R_{\theta} p_{j}$ are linear combinations of the Zernike polynomials of (1.6), and so belong to $\mathcal{P}_{n}$ and are orthogonal to each other for different values of $j$. In view of the rotational invariance of the inner product, it therefore suffices to show that

$$
\left\langle p_{j}, R_{\theta} p_{j}\right\rangle=\cos ((n-2 j) \theta)
$$

Using the change of variables $x=r^{2}$, we compute the norms of the Zernike polynomials

$$
\begin{aligned}
h_{j} & :=\left\||z|^{2 n-j} L_{j}^{n-2 j+\beta}\left(|z|^{2}\right)\right\|^{2}=\left\|z^{2 n-j} L_{j}^{n-2 j+\beta}\left(|z|^{2}\right)\right\|^{2}=\left\|\bar{z}^{2 n-j} L_{j}^{n-2 j+\beta}\left(|z|^{2}\right)\right\|^{2} \\
& =2 \pi \int_{0}^{\infty}\left(L_{j}^{n-2 j+\beta}\left(r^{2}\right)\right)^{2} r^{4 n-2 j} r^{2 \beta} e^{-r^{2}} r d r=2 \pi \int_{0}^{\infty}\left(L_{j}^{n-2 j+\beta}(x)\right)^{2} x^{2 n-j+\beta} e^{-x} \frac{d x}{2} \\
& =\pi \frac{\Gamma(n-2 j+\beta+j+1)}{j !}=\pi \frac{\Gamma(\beta+n-j+1)}{j !} .
\end{aligned}
$$


For $j \neq \frac{n}{2}$, there are two Zernike polynomials which are orthogonal to each other, giving $\left\|\left(z^{n-2 j}+\bar{z}^{n-2 j}\right) L_{j}^{n-2 j+\beta}\left(|z|^{2}\right)\right\|^{2}=\left\|z^{n-2 j} L_{j}^{n-2 j+\beta}\left(|z|^{2}\right)\right\|^{2}+\left\|\bar{z}^{n-2 j} L_{j}^{n-2 j+\beta}\left(|z|^{2}\right)\right\|^{2}=2 h_{j}$, and for $j=\frac{n}{2}$ (when $n$ even) there is just one Zernike polynomial, so that

$$
\left\|\left(z^{n-2 j}+\bar{z}^{n-2 j}\right) L_{j}^{n-2 j+\beta}\left(|z|^{2}\right)\right\|=2\left\|L_{\frac{n}{2}}^{n-2 j+\beta}\left(|z|^{2}\right)\right\|=2 \sqrt{h_{\frac{n}{2}}} .
$$

These together with (2.4) show that the $p_{j}$ have unit norm.

Let $\xi:=e^{i \theta}$, then up to a constant scalar, which is independent of $\theta$, the inner product $\left\langle p_{j}, R_{\theta} p_{j}\right\rangle$ is given by

$$
\begin{aligned}
\left\langle\left(z^{n-2 j}+\bar{z}^{n-2 j}\right) L_{j}^{n-2 j+\beta}\left(|z|^{2}\right),\left((\xi z)^{n-2 j}+(\overline{\xi z})^{n-2 j}\right) L_{j}^{n-2 j+\beta}\left(|\xi z|^{2}\right)\right\rangle \\
=\left\langle\left(z^{n-2 j}+\bar{z}^{n-2 j}\right) L_{j}^{n-2 j+\beta}\left(|z|^{2}\right),\left(\xi^{n-2 j} z^{n-2 j}+\bar{\xi}^{n-2 j} \bar{z}^{n-2 j}\right) L_{j}^{n-2 j+\beta}\left(|z|^{2}\right)\right\rangle \\
=\xi^{n-2 j}\left\|z^{n-2 j} L_{j}^{n-2 j+\beta}\left(|z|^{2}\right)\right\|^{2}+\bar{\xi}^{n-2 j}\left\|\bar{z}^{n-2 j} L_{j}^{n-2 j+\beta}\left(|z|^{2}\right)\right\|^{2} \\
=2 \operatorname{Re}\left(\xi^{n-2 j} h_{j}\right)=2 h_{j} \cos ((n-2 j) \theta) .
\end{aligned}
$$

Since $\left\langle p_{j}, R_{0} p_{j}\right\rangle=\left\|p_{j}\right\|^{2}=1$, this completes the result.

The above result gives an orthogonal decomposition of $\mathcal{P}_{n}$

$$
\mathcal{P}_{n}=\bigoplus_{0 \leq j \leq \frac{n}{2}} V_{j}, \quad V_{j}:=\operatorname{span}\left\{R_{\theta} p_{j}: 0 \leq \theta \leq 2 \pi\right\}
$$

into $\mathrm{SO}(2)$-invariant subspaces $V_{j}$, which have dimensions

$$
\operatorname{dim}\left(V_{j}\right)= \begin{cases}2, & j \neq \frac{n}{2} \\ 1, & j=\frac{n}{2}\end{cases}
$$

We can now give our orthonormal basis.

Theorem 2.5 (Orthonormal basis). Let $\theta_{j} \in[0,2 \pi], 0 \leq j \leq \frac{n}{2}$, and define $p: \mathbb{R}^{2} \rightarrow \mathbb{R}$ by

$$
p:=\sum_{0 \leq j \leq \frac{n}{2}} \sqrt{\frac{2-\delta_{j, \frac{n}{2}}}{n+1}} R_{\theta_{j}} p_{j}, \quad \theta_{j} \in[0,2 \pi] .
$$

Then $\left\{R_{\frac{\ell \pi}{n+1}} p\right\}_{\ell=0}^{n}$ is an orthonormal basis for $\mathcal{P}_{n}$. In particular, for $\theta_{j}=0$, we have

$$
p(x, y)=\frac{1}{\sqrt{\pi}} \frac{1}{\sqrt{n+1}} \sum_{0 \leq j \leq \frac{n}{2}} \frac{1}{1+\delta_{j, \frac{n}{2}}} \frac{\sqrt{j !}}{\sqrt{\Gamma(\beta+n-j+1)}}\left(z^{n-2 j}+\bar{z}^{n-2 j}\right) L_{j}^{n-2 j+\beta}\left(|z|^{2}\right) .
$$


Proof: Let $\mu:=e^{\frac{i \pi}{n+1}}$ be a primitive $2(n+1)$-th root of unity, and $\omega:=\mu^{2}$ a primitive $(n+1)$-th root. For $\ell$ and $m$ integers, the orthogonality property $(2.3)$, gives

$$
\begin{aligned}
\left\langle R_{\frac{\ell \pi}{n+1}} p, R_{\frac{m \pi}{n+1}} p\right\rangle & =\left\langle\sum_{0 \leq j \leq \frac{n}{2}} \sqrt{\frac{2-\delta_{j, \frac{n}{2}}}{n+1}} R_{\theta_{j}+\frac{\ell \pi}{n+1}} p_{j}, \sum_{0 \leq k \leq \frac{n}{2}} \sqrt{\frac{2-\delta_{k, \frac{n}{2}}}{n+1}} R_{\theta_{k}+\frac{m \pi}{n+1}} p_{k}\right\rangle \\
& =\sum_{0 \leq j \leq \frac{n}{2}} \frac{2-\delta_{j, \frac{n}{2}}}{n+1}\left\langle R_{\theta_{j}+\frac{\ell \pi}{n+1}} p_{j}, R_{\theta_{j}+\frac{m \pi}{n+1}} p_{k}\right\rangle \\
& =\sum_{0 \leq j \leq \frac{n}{2}} \frac{2-\delta_{j, \frac{n}{2}}}{n+1} \cos \left((n-2 j)(\ell-m) \frac{2 \pi}{2(n+1)}\right) \\
& =\frac{1}{n+1} \sum_{0 \leq j \leq \frac{n}{2}} \frac{2-\delta_{j, \frac{n}{2}}}{2}\left(\mu^{(n-2 j)(\ell-m)}+\bar{\mu}^{(n-2 j)(\ell-m)}\right) \\
& =\frac{1}{n+1} \sum_{0 \leq j \leq \frac{n}{2}} \frac{2-\delta_{j, \frac{n}{2}}}{2}(-1)^{\ell-m}\left(\omega^{-(\ell-m) j}+\omega^{(\ell-m) j}\right) \\
& =\frac{(-1)^{\ell-m}}{n+1} \sum_{j=0}^{n}\left(\omega^{\ell-m}\right)^{j} .
\end{aligned}
$$

Since $\omega^{\ell-m}$ is an $(n+1)$-th root of unity, the last sum above is zero, except for the case $\ell \equiv m \quad(\bmod n+1)$, when it is 1 for $m=\ell$.

As the proof indicates, in (2.2) one could also allow $R_{\theta_{j}} p_{j}$ to be multiplied by \pm 1 (or unit modulus complex scalars), and using (2.3) one could determine how many of these polynomials are different. It follows from [VW05:Th.6.18] that these are all such polynomials.

This expansion is of a similar type to that of Logan and Schepp [LS75]. They showed that the Legendre polynomials (constant weight) on the disc

$$
\mathbb{D}:=\left\{(x, y) \in \mathbb{R}^{2}: x^{2}+y^{2} \leq 1\right\}
$$

have an orthonormal basis given by the $n+1$ polynomials

$$
p_{\ell}(x, y):=\frac{1}{\sqrt{\pi}} U_{n}\left(x \cos \frac{\ell \pi}{n+1}+y \sin \frac{j \pi}{n+1}\right), \quad \ell=0, \ldots, n,
$$

where $U_{n}$ is the $n$-th Chebyshev polynomial of the second kind, i.e., $\left\{R_{\frac{\ell \pi}{n+1}} p_{0}\right\}_{\ell=0}^{n}$ is an orthonormal basis.

The polynomial $p_{0}$ above is a function of $x$ only, and so is a ridge function. A simple calculation shows that the generalised Hermite polynomials $\mathcal{P}_{4}$ contain ridge functions if and only if

$$
4 m_{1}^{2} m_{3}-3 m_{1} m_{2}^{2}-m_{0} m_{2} m_{3}=0 \quad \Longleftrightarrow \quad \beta=0 .
$$

For Hermite polynomials, (1.4) gives a ridge function $h_{n}$ in $\mathcal{P}_{n}$, namely

$$
h_{n}(x, y):=H_{n}(x) .
$$


However, the rotates of this function do not give an orthonormal basis, since

$$
\frac{\left\langle h_{n}, R_{\frac{\ell \pi}{n+1}} h_{n}\right\rangle}{\left\langle h_{n}, h_{n}\right\rangle}=\left(\cos \frac{\ell \pi}{n+1}\right)^{n}, \quad 0 \leq \ell \leq n .
$$

It is therefore not possible to choose the polynomial $p$ of (2.6) to be a ridge function.

From (1.3), it follows that

$$
(-1)^{n} R_{\frac{\ell \pi}{n+1}} p=R_{\frac{\ell \pi}{n+1}} R_{\pi} p=R_{\frac{\ell \pi}{n+1}+\frac{\pi(n+1)}{n+1}} p=R_{\frac{2 \pi}{2(n+1)}}^{\ell+n+1} p
$$

so that Theorem 2.5 gives

$$
\begin{aligned}
f & =\sum_{\ell=0}^{n}\left\langle f, R_{\frac{\ell \pi}{n+1}} p\right\rangle R_{\frac{\ell \pi}{n+1}} p \\
& =\frac{1}{2} \sum_{\ell=0}^{n}\left\langle f, R_{\frac{2 \pi}{2(n+1)}}^{\ell} p\right\rangle R_{\frac{2 \pi}{2(n+1)}}^{\ell} p+\frac{1}{2} \sum_{\ell=0}^{n}\left\langle f, R_{\frac{2 \pi}{2(n+1)}}^{\ell+n+1} p\right\rangle R_{\frac{2 \pi}{2(n+1)}}^{\ell+n+1} p \\
& =\frac{1}{2} \sum_{\ell=0}^{2 n+1}\left\langle f, R_{\frac{2 \pi}{2(n+1)}}^{\ell} p\right\rangle R_{\frac{2 \pi}{2(n+1)}}^{\ell} p, \quad \forall f \in \mathcal{P}_{n},
\end{aligned}
$$

i.e., $f \in \mathcal{P}_{n}$ can be written as a sum of its projections onto the rotates $\left\{R_{\frac{2 \pi \ell}{2(n+1)}} p\right\}_{\ell=0}^{2 n+1}$. We now show that a continuous version of this also holds.

Corollary 2.9 (Integral expansion). Let $p \in \mathcal{P}_{n}$ be given by (2.6). Then

$$
f=\frac{n+1}{2 \pi} \int_{0}^{2 \pi}\left\langle f, R_{\theta} p\right\rangle R_{\theta} p d \theta, \quad \forall f \in \mathcal{P}_{n} .
$$

Proof: $\quad$ For any $f \in \mathcal{P}_{n}$ and $(x, y) \in \mathbb{R}^{2}$, the function $T: \theta \mapsto\left\langle f, R_{\theta} p\right\rangle R_{\theta} p(x, y)$ is a trigonometric polynomial of degree $2 n$. Hence we can find its integral by using the quadrature formula involving $2(n+1)(>2 n)$ equally spaced nodes

$$
\begin{aligned}
\frac{1}{2 \pi} \int_{0}^{2 \pi}\left\langle f, R_{\theta} p\right\rangle R_{\theta} p(x, y) d \theta & =\frac{1}{2 \pi} \int_{0}^{2 \pi} T(\theta) d \theta=\frac{1}{2(n+1)} \sum_{\ell=0}^{2 n+1} T\left(\frac{2 \pi \ell}{2(n+1)}\right) \\
& =\frac{1}{2(n+1)} \sum_{\ell=0}^{2 n+1}\left\langle f, R_{\frac{2 \pi \ell}{2(n+1)}} p\right\rangle R_{\frac{2 \pi \ell}{2(n+1)}} p(x, y) \\
& =\frac{1}{n+1} f(x, y),
\end{aligned}
$$

with the last equality following from (2.8). This gives (2.10). 


\section{References}

[DX01] C. F. Dunkl and Y. Xu, "Orthogonal polynomials of several variables", Cambridge University Press, Cambridge, 2001.

[LS75] B. F. Logan and L. A. Shepp, Optimal reconstruction of a function from its projections, Duke Math. J. 42 (1975), 645-659.

[VW05] R. Vale and S. Waldron, Tight frames and their symmetries, Constr. Approx. 21 (2005), 83-112.

[W01] A. Wünsche, Hermite and Laguerre 2D polynomials, J. Comput. Appl. Math. 133 (2001), 665-678. 